\title{
Fractional moments of the Riemann zeta-function
}

\author{
by
}

K. RamaChandRA (Bombay and Bangalore)

To Professor Kannan Soundararajan on his twenty-third birthday

1. Introduction. The object of this paper is to prove the following theorem.

THEOREM 1. Let $k=p q^{-1}$ where $p$ and $q$ are integers subject to $1 \leq$ $p \leq q(\log (q+1))^{-1 / 2}$. Let $T \geq H \geq C_{0} \log \log \left(T^{k}+100\right)$ where $C_{0}>0$ is a certain large absolute constant. Then for $T \geq 10$, we have

$$
\frac{1}{H} \int_{T}^{T+H}|\zeta(1 / 2+i t)|^{2 k} d t>C_{1}(\log H)^{k^{2}}
$$

where $C_{1}>0$ is a certain absolute constant $\left(C_{0}\right.$ and $C_{1}$ are effective $)$.

Re mark 1. In place of $(\log (q+1))^{-1 / 2}$ we can have $C_{2}(\log (q+1))^{-1 / 2}$ where $C_{2}>0$ is any absolute constant. Then $C_{0}$ and $C_{1}$ depend on $C_{2}$.

Remark 2. The previous history of the theorem is as follows. First, E. C. Titchmarsh considered the case $H=T$, and $k$ any positive integer, of (1) and proved that

$$
\limsup _{T \rightarrow \infty}\left((\mathrm{LHS})(\mathrm{RHS})^{-1}\right)>0 .
$$

Next I considered the case where $k$ is half of any positive integer and proved (1) (however with $C_{1}$ depending possibly on $k$ ). Next D. R. Heath-Brown [1] considered the case $H=T$ and $k$ any positive rational number and proved (1) (however with $C_{1}$ depending possibly on $k$ ). Next M. Jutila [4] considered the case $H=T$ and $k=q^{-1}$ and proved (1) with $C_{1}$ independent of $k$. For all these references see also my book [6]. Two other excellent reference books are [7] and [2].

Remark 3. We use only "Euler product" in the proof of Theorem 1 and so its analogue goes through for $L$-functions of algebraic number fields, Ramanujan's zeta-function and so on. 


\section{Some preliminaries to the proof}

Theorem 2 (H. L. Montgomery and R. C. Vaughan [5]). Let $H>0$, $N \geq 1$ be an integer, and $a_{1}, \ldots, a_{N}$ any $N$ complex numbers. Then

$$
\int_{0}^{H}\left|\sum_{n \leq N} a_{n} n^{i t}\right|^{2} d t=\sum_{n \leq N}(H+O(n))\left|a_{n}\right|^{2} .
$$

Moreover, the $O$-constant is absolute.

R e m a r k 1 . Montgomery and Vaughan obtained an economical $O$-constant (see [6], p. 21, for a proof with some absolute constant).

Remark 2. We use Theorem 2 with $N$ something like $N=H^{7 / 8}(H \geq$ 10) and for this choice there are much simpler methods of proving what we want.

Theorem 3 (K. Ramachandra [6]). Let $z=x+i y$ be a complex variable with $|x| \leq 1 / 4$. Then:

(a) $\left|\exp \left((\sin z)^{2}\right)\right| \leq 2$ for all $y$.

(b) If $|y| \geq 2$ we have

$$
\left|\exp \left((\sin z)^{2}\right)\right| \leq 2(\exp \exp |y|)^{-1} .
$$

Proof. See [6], p. 38.

TheOrem 4. Let $q>0$ and $a>0$ be real numbers and $n$ any positive integer. Consider the rectangle defined by

$$
0 \leq x \leq\left(2^{n}+1\right) a, \quad-R \leq y \leq R .
$$

Let $f(z)$ and $\varphi(z)$ be two functions analytic inside this rectangle and let $|f(z)|$ and $|\varphi(z)|$ be continuous on its boundary. Let

$$
I_{x}=\int_{-R}^{R}|\varphi(z)| \cdot|f(z)|^{1 / q} d y
$$

and let

$$
Q(\alpha)=\max \left(|\varphi(z)| \cdot|f(z)|^{1 / q}\right)
$$

taken over $0 \leq x \leq \alpha, y= \pm R$. Then with $b_{n}=2^{n}+1$ we have

$$
I_{a} \leq\left(I_{0}+U\right)^{1 / 2}\left(I_{a}+U\right)^{1 / 2-2^{-n-1}}\left(I_{a b_{n}}+U\right)^{2^{-n-1}}
$$

where $U=2^{2(n+1)} Q\left(a b_{n}\right) a$.

Proof. See [6], p. 97. (Here we have replaced the interval $(0, R)$ by $(-R, R)$ and the number $q$ by $1 / q$.)

Theorem 5. Let $w=u+i v$ and $s=\sigma+i$ be two complex variables,

$$
K(w)=\exp \left(\left(\sin \frac{w}{8 A}\right)^{2}\right)
$$


where $A>0$ is a large constant, and let

$$
f(s, w)=(K(w))^{q} f_{0}(s+w)
$$

where $q(>0)$ is any real number. Let $K(w)$ and $f_{0}(s+w)$ satisfy the conditions of Theorem 4 with

$$
\varphi(z)=K(z+a) \quad \text { and } \quad f(z)=f_{0}(s+z+a) .
$$

Then if we take $R=\tau$ we have, with $b_{n}=2^{n}+1$,

$$
\begin{aligned}
\int_{|v| \leq \tau}|f(s, w)|_{u=0}^{1 / q} d v \leq & \left(\int_{|v| \leq \tau}|f(s, w)|_{u=-a}^{1 / q} d v+H^{-10}\right)^{1 / 2} \\
& \times\left(\int_{|v| \leq \tau}|f(s, w)|_{u=0}^{1 / q} d v+H^{-10}\right)^{1 / 2-2^{-n-1}} \\
& \times\left(\int_{|v| \leq \tau}|f(s, w)|_{u=a b_{n}-a}^{1 / q} d v+H^{-10}\right)^{2^{-n-1}}
\end{aligned}
$$

provided $U \leq H^{-10}$.

THEOREM 6. If the conditions of Theorem 5 are satisfied uniformly for $t$ belonging to an interval $B \leq t \leq B+H_{1}$ with $0 \leq H_{1} \leq H$, we have (2) with $\int_{|v| \leq \tau} \ldots d v$ replaced by $\int_{(t)} \int_{|v| \leq \tau} \ldots d v d t$ and $H^{-10}$ replaced by $H^{-9}$. Moreover, if

$$
\int_{(t)} \int_{|v| \leq \tau}|f(s, w)|_{u=0}^{1 / q} d v d t \geq H^{-9}
$$

then

(4)

$$
\begin{aligned}
\int_{(t)} \int_{|v| \leq \tau}|f(s, w)|_{u=0}^{1 / q} d v d t & \\
\leq & 2\left(\int_{(t)} \int_{|v| \leq \tau}|f(s, w)|_{u=-a}^{1 / q} d v d t+H^{-9}\right)^{2^{n} /\left(2^{n}+1\right)} \\
& \times\left(\int_{(t)} \int_{|v| \leq \tau}|f(s, w)|_{u=a 2^{n}}^{1 / q} d v d t+H^{-9}\right)^{1 /\left(2^{n}+1\right)} .
\end{aligned}
$$

Pro of. Under the assumption (3) we can replace the second factor on the RHS of (2) by

$$
\left(2 \int_{(t)} \int_{|v| \leq \tau}|f(s, w)|_{u=0}^{1 / q} d v d t\right)^{1 / 2-2^{-n-1}} .
$$

This gives Theorem 6 . 
THEOREM 7. LHS of (4) is

$$
\gg \int_{B+\tau}^{B+H_{1}-\tau}\left|f_{0}(\sigma+i t)\right|^{1 / q} d t
$$

where the interval for $t$ is $\left(B, B+H_{1}\right)$, provided $2 \tau \leq H_{1}$. Also for any $u$ on RHS of (4) we have

$$
\int_{(t)} \int_{|v| \leq \tau} \ldots d v d t \ll \int_{B-\tau}^{B+H_{1}+\tau}\left|f_{0}(\sigma+i t+u)\right|^{1 / q} d t .
$$

Proof. LHS of (4) equals

$$
\begin{aligned}
& \int_{B}^{B+H_{1}} \int_{|v| \leq \tau} K(i v)\left|f_{0}(\sigma+i t+i v)\right|^{1 / q} d v d t \\
& =\int_{(v)} K(i v)\left(\int_{(t)} \ldots d t\right) d v=\int_{(v)} K(i v)\left(\int_{B+v}^{B+H_{1}-v} \ldots d t\right) d v \\
& \quad>\int_{(v)} K(i v)\left(\int_{B+\tau}^{B+H_{1}-\tau} \ldots d t\right) d v=\left(\int_{(v)} K(i v) d v\right)\left(\int_{B+\tau}^{B+H_{1}-\tau} \ldots d t\right)
\end{aligned}
$$

and this proves the first part of Theorem 7 . The proof of the second part is similar.

R e mark. Theorems 6 and 7 are stated here for the first time although they are already implicitly contained in [6]. These are new versions of the convexity.

Theorem 8 (D. R. Heath-Brown and M. Jutila [1], [4]). Let $k(>0)$ be any real number. Then for $1 / 2<\sigma \leq 2$, we have

$$
\sum_{n=1}^{\infty}\left(d_{k}(n)\right)^{2} n^{-2 \sigma} \leq(\zeta(2 \sigma))^{k^{2}} \leq A_{1}^{k^{2}}(\sigma-1 / 2)^{-k^{2}},
$$

where $A_{1}>0$ is an absolute constant. (Here $d_{k}(n)$ are defined as usual by $(\zeta(s))^{k}=\sum_{n=1}^{\infty} d_{k}(n) n^{-s}$, Re $s \geq 2$.) Also let $N \geq 2$ and $0<k \leq 1$. Then there exists an absolute constant $A_{2}>0$ for which

$$
\sum_{n \leq N}\left(d_{k}(n)\right)^{2} n^{-2 \sigma} \geq A_{2}(\sigma-1 / 2)^{-k^{2}}
$$

provided

$$
1 / 2+A_{3}(\log N)^{-1} \leq \sigma \leq 2,
$$

with an absolute constant $A_{3}(>0)$ which depends only on $A_{2}$. 
Remark. We can allow any (absolute) constant upper bound for $k$ and still prove the second part of the theorem.

Proof of Theorem 8. The first part follows from the inequality $\left(d_{k}(n)\right)^{2} \leq d_{k^{2}}(n)$. The second part (due essentially to D. R. HeathBrown [1]) can be proved as follows. For all $\delta>0,(1+\delta) / 2 \leq \sigma<2$, we have

$$
\begin{aligned}
\sum_{n \leq N}\left(d_{k}(n)\right)^{2} n^{-2 \sigma} & \geq \sum_{n=1}^{\infty}\left(d_{k}(n)\right)^{2}|\mu(n)| n^{-2 \sigma}\left(1-\left(\frac{n}{N}\right)^{\delta}\right) \\
& \geq \prod_{p}\left(1+\frac{k^{2}}{p^{2 \sigma}}\right)-N^{-\delta} A_{1}\left(\sigma-\frac{\delta}{2}-\frac{1}{2}\right)^{-k^{2}} .
\end{aligned}
$$

(Here and in the next line $p$ is a symbol running over all primes and it should not be confused with $p$ in Theorem 1.) Here the product over $p$ is

$$
\left[\exp \sum_{p}\left\{\log \left(1+\frac{k^{2}}{p^{2 \sigma}}\right)-k^{2} \log \left(\frac{1}{1-p^{-2 \sigma}}\right)\right\}\right](\zeta(2 \sigma))^{k^{2}}
$$

which exceeds $A_{4}(\sigma-1 / 2)^{-k^{2}}$. Thus

$$
\sum_{n \leq N} d_{k}(n) n^{-2 \sigma} \geq A_{4}\left(\sigma-\frac{1}{2}\right)^{-k^{2}}\left\{1-\frac{A_{1}}{A_{4}} N^{-\delta}\left(\frac{\sigma-1 / 2}{\sigma-(1+\delta) / 2}\right)^{k^{2}}\right\} .
$$

Here we set $\delta=\sigma-1 / 2$ and obtain for the RHS the lower bound

$$
\begin{aligned}
A_{4}\left(\sigma-\frac{1}{2}\right)^{-k^{2}}\left\{1-\frac{A_{1}}{A_{4}} N^{-\delta} 2^{k^{2}}\right\} & \geq A_{4}\left(\sigma-\frac{1}{2}\right)^{-k^{2}}\left(1-\frac{2 A_{1}}{A_{4}} N^{1 / 2-\sigma}\right) \\
& \geq A_{4}\left(\sigma-\frac{1}{2}\right)^{-k^{2}}\left(1-\frac{2 A_{1}}{A_{4}} e^{-A_{3}}\right) \\
& =\left(A_{4}-2 A_{1} e^{-A_{3}}\right)\left(\sigma-\frac{1}{2}\right)^{-k^{2}}
\end{aligned}
$$

and this proves the second part of Theorem 8 .

THEOREM 9. Let $f(z)$ be analytic in $|z| \leq r$. Then for any real $k>0$, we have

$$
|f(0)|^{k} \leq \frac{1}{\pi r^{2}} \int_{|z| \leq r} \int|f(z)|^{k} d x d y .
$$

Proof. See [6], p. 34.

3. Proof of Theorem 1 (first step). The main object of this section is to prove the following theorem. (From now on we assume that $k=p / q$ where $p$ and $q$ are integers subject to $1 \leq p \leq q(\log (q+1))^{-1 / 2}$.) 
TheOREM 10. Let $T \geq H$ and $H$ exceed a certain large positive absolute constant. Then

$$
\max _{\sigma \geq 1 / 2+q(\log H)^{-1}}\left(\frac{1}{H} \int_{T}^{T+H}|\zeta(\sigma+i t)|^{2 k} d t\right) \geq C_{2}(\log H)^{k^{2}}
$$

where $C_{2}>0$ is an absolute constant (not to be confused with $C_{2}$ of Remark 1 below Theorem 1).

Remark. If $q \geq(\log H)^{1 / 100}$, then $(\log H)^{k^{2}}$ lies between two positive constants and also for $\sigma \geq 2$,

$$
|\zeta(\sigma+i t)|^{-1} \leq \zeta(2)<1+\sum_{n=2}^{\infty}(n(n-1))^{-1}=2
$$

and so $|\zeta(\sigma+i t)| \geq 1 / 2$. Hence $|\zeta(\sigma+i t)|^{2 k} \geq 2^{-4}=1 / 16$. Thus Theorem 10 is obvious in this case.

From now on till the end of this section we assume that $1 \leq q \leq$ $(\log H)^{1 / 100}$ and that for all $\sigma \geq 1 / 2+q(\log H)^{-1}$, we have

$$
\frac{1}{H} \int_{T}^{T+H}|\zeta(\sigma+i t)|^{2 k} d t<C_{2}(\log H)^{k^{2}}
$$

where $C_{2}(>0)$ is a small constant. (Finally, we arrive at a contradiction.)

Note that assuming (6) it suffices to either get a contradiction or to prove Theorem 10 with

$$
\frac{1}{H} \int_{T}^{T+H}|\zeta(\sigma+i t)|^{2 k} d t
$$

replaced by

$$
\frac{1}{H-H_{0}} \int_{T+H_{0}}^{T+H-H_{0}}|\zeta(\sigma+i t)|^{2 k} d t
$$

(and $C_{2}$ replaced by $C_{2}^{*}$ (a small positive constant)) where $H_{0}$ lies between two (small absolute) positive constant multiples of $H$. Note also that the maximum over any region is greater than or equal to the maximum taken over a sub-region.

Lemma 1. For $\sigma \geq 1 / 2+(q+2)(\log H)^{-1}, T+1 \leq t \leq T+H-1$, we have

$$
|\zeta(s)|^{2 k} \leq H^{2} .
$$

Proof. Take the circle $|z| \leq(\log H)^{-1}$, apply Theorem 9 to $f(z)=$ $\zeta(s+z)$ and $(7)$ follows. 
We next apply Theorems 5, 6 and 7 with

$$
f_{0}(z)=(\zeta(z))^{2 p}-\left(P_{N}(z)\right)^{2 q}
$$

where

$$
P_{N}(z)=\sum_{n \leq N} d_{k}(n) n^{-z}, \quad N=H^{7 / 8} .
$$

From now on we assume $\sigma \geq 1 / 2+(q+2)(\log H)^{-1}$.

Lemma 2. For $\mathrm{H}_{2}$ with $0 \leq 2 \mathrm{H}_{2} \leq H$, the quantity

$$
\int_{T+H_{2}}^{T+H-H_{2}}\left|(\zeta(\sigma+i t))^{2 p}-\left(P_{N}(\sigma+i t)\right)^{2 q}\right|^{q^{-1}} d t
$$

lies between

$$
\int_{T+H_{2}}^{T+H-H_{2}}\left|P_{N}(\sigma+i t)\right|^{2} d t-C_{2} H(\log H)^{k^{2}}
$$

and

$$
\int_{T+H_{2}}^{T+H-H_{2}}\left|P_{N}(\sigma+i t)\right|^{2} d t+C_{2} H(\log H)^{k^{2}} .
$$

Proof. For any two complex numbers $z_{1}$ and $z_{2}$ we show that

$$
\left|z_{1}\right|^{q^{-1}}-\left|z_{2}\right|^{q^{-1}} \leq\left|z_{1}-z_{2}\right|^{q^{-1}} \leq\left|z_{1}\right|^{q^{-1}}+\left|z_{2}\right|^{q^{-1}} .
$$

The latter inequality follows on raising both sides to the power $q$ and using $\left|z_{1}\right|+\left|z_{2}\right| \geq\left|z_{1}-z_{2}\right|$. The former is similar: we have to use $\left|z_{1}\right| \leq\left|z_{2}\right|+$ $\left|z_{1}-z_{2}\right|$.

LEMMA 3. If $H_{2} \leq(1000)^{-1} H$, the quantity $\int_{T+H_{2}}^{T+H-H_{2}}\left|P_{N}(\sigma+i t)\right|^{2} d t$ lies between $C_{3} H(\sigma-1 / 2)^{-k^{2}}$ and $C_{4} H(\sigma-1 / 2)^{-k^{2}}$, where $C_{3}>0$ and $C_{4}>0$ are absolute constants (independent of $C_{2}$ ) provided $\sigma \leq 2$.

Proof. Apply Theorems 2 and 8.

LEMMA 4. Let $\sigma_{0}=1 / 2+10 q(\log H)^{-1}, a=D q(\log H)^{-1}, s=\sigma_{0}+a+i t$, where $D>0$ is any large absolute constant and $T+H_{3} \leq t \leq T+H-H_{3}$, where $H_{3}$ is a small positive constant multiple of $H$. Then with $\tau$ equal to a small positive constant multiple of $H$, we have

$$
\int_{(t)} \int_{|v| \leq \tau}|f(s, w)|_{u=0}^{1 / q} d v d t \geq H^{-9},
$$

$$
\int_{(t)} \int_{|v| \leq \tau}|f(s, w)|_{u=0}^{1 / q} d v d t \geq C_{5} H(\log H)^{k^{2}} D^{-k^{2}},
$$




$$
\int_{(t)} \int_{|v| \leq \tau}|f(s, w)|_{u=-a}^{1 / q} d v d t+H^{-9} \leq C_{6} H(\log H)^{k^{2}}
$$

and

$$
\int_{(t)} \int_{|v| \leq \tau}|f(s, w)|_{u=a 2^{n}}^{1 / q} d v d t+H^{-9} \leq C_{7} H^{1-a 2^{n} /(100 q)},
$$

where $a 2^{n}$ lies between 10 and 20 . Here $C_{5}, C_{6} \geq 1$ and $C_{7} \geq 1$ are positive constants ( since $C_{2}$ can be fixed to be small) and $D^{-k^{2}}$ exceeds a certain positive absolute constant times $C_{2}$ for the validity of (14).

Proof. This follows from Theorem 3 and assumption (6) and its consequence (7). Note that $q^{k^{2}}$ lies between two absolute positive constants. We give some details in proving (16). We have

$$
|f(s, w)|_{u=a 2^{n}}^{1 / q} \leq|K(w+a)| \cdot\left|(\zeta(s+w+a))^{2 p}-\left(P_{N}(s+w+a)\right)^{2 q}\right|_{u=a 2^{n}}^{1 / q}
$$

with $N=H^{7 / 8}$ and

Also

$$
|K(w+a)| \ll\left(\exp \exp \frac{|v|}{8 A}\right)^{-1} .
$$

$$
\begin{aligned}
& \left|(\zeta(s+w+a))^{2 p}-\left(P_{N}(s+w+a)\right)^{2 q}\right|_{u=a 2^{n}} \\
& \quad=\left|\left((\zeta(s+w+a))^{p / q}\right)^{2 q}-\left(P_{N}(s+w+a)\right)^{2 q}\right|_{u=a 2^{n}} \\
& \quad \leq \mid(\zeta h+w+w+a))^{p / q}-\left.P_{N}(s+w+a)\right|_{u=a 2^{n}}(100)^{2 p+2 q} \\
& \quad \ll\left(\sum_{n \geq N} n^{-10}\right)(100)^{2 p+2 q} \ll N^{-9}(100)^{2 p+2 q}=H^{-63 / 8}(100)^{2 p+2 q} .
\end{aligned}
$$

Thus

Finally

$$
|f(s, w)|_{u=a 2^{n}}^{1 / q} \ll\left(\exp \exp \frac{|v|}{8 A}\right)^{-1} H^{-63 /(8 q)}
$$

$$
\frac{63}{8 q} \geq \frac{a 2^{n}}{100 q} \quad \text { since } a 2^{n} \leq \frac{6300}{8} .
$$

These calculations prove (16).

Lemma 5. We have

$$
\begin{aligned}
C_{5} D^{-4} H(\log H)^{k^{2}} & \\
\leq & 2\left(C_{6} H(\log H)^{k^{2}}\right)^{2^{n} /\left(2^{n}+1\right)}\left(C_{7} H^{1-2^{n} a /(100 q)}\right)^{1 /\left(2^{n}+1\right) .}
\end{aligned}
$$

Proof. This follows from Theorem 6 and Lemma 4 . 
LEMma 6. We have

$$
H^{-2^{n} a\left(2^{n}+1\right)^{-1}(100 q)^{-1}} \leq H^{-D(200 \log H)^{-1}} \leq e^{-D / 200}
$$

and $\frac{1}{2}\left(2^{n}+1\right) \leq 2^{n}<2^{n}+1$.

Pr o of. Trivial.

Lemmas 5 and 6 end up with the contradiction

$$
C_{5} D^{-4} \leq 2 C_{6} C_{7} e^{-D / 200}
$$

provided we fix $C_{2}=D^{-100}$ and choose $D$ to be large enough. Thus Theorem 10 is completely proved.

4. Deduction of Theorem 1 from Theorem 10 (second and final step). Actually our proof of Theorem 10 with a trivial modification gives

$$
\max _{\sigma \geq 1 / 2+q(\log H)^{-1}}\left(\frac{1}{H} \int_{T+H_{4}}^{T+H-H_{4}}|\zeta(\sigma+i t)|^{2 k} d t\right)>C_{8}(\log H)^{k^{2}}
$$

where $C_{8}>0$ is absolute and $H_{4}$ is a small (absolute) positive constant times $H$. We first prove

Theorem 11. If $q \geq(\log H)^{1 / 100}$ then (1) is true.

Pro of. We argue as we did after proving Lemma 1 but with $f_{0}(z)=\zeta(z)$, $\sigma_{0}=1 / 2, a=10, n=2$. Note that $(\log H)^{k^{2}}$ lies between two absolute positive constants. We use $|\zeta(\sigma+i t)| \ll t^{1 / 2}$ uniformly for $\sigma \geq 1 / 2, t \geq 10$ and we see that we need the condition

$$
\left(\exp \exp \left(C_{9} H\right)\right)^{-1} T^{k} \leq H^{-11} \quad\left(C_{9}>0 \text { is an absolute constant }\right),
$$

which is precisely the condition $H \geq C_{0} \log \log \left(T^{k}+100\right)$ of Theorem 1 . We need the condition $H \leq T$ for the bound on $|\zeta(\sigma+i t)|$ mentioned above.

We only have to prove the following theorem.

TheOREM 12. Let $q \leq(\log H)^{1 / 100}$. Then (1) is true.

Proof. We use (17). We fix $a$ to be the largest $\sigma \leq 2$ with the property

$$
\frac{1}{H} \int_{T+H_{4}}^{T+H-H_{4}}|\zeta(\sigma+i t)|^{2 k} d t>C_{8}(\log H)^{k^{2}}
$$

and $\sigma_{0}$ to be $1 / 2$. We argue as before with $f_{0}(z)=\zeta(z)$, where $n$ is such that $a 2^{n}$ lies between 10 and 20 . Note that in this case

$$
\int_{(t)} \int_{|v| \leq \tau}|f(s, w)|_{u=a 2^{n}}^{1 / q} d v d t+H^{-9}
$$


does not exceed an absolute constant times $H$. We use $|\zeta(\sigma+i t)| \ll t^{1 / 2}$ for $\sigma \geq 1 / 2$ and $t \geq 10$ and we see that we need the condition

$$
\left(\exp \exp \left(C_{10} H\right)\right)^{-1} T^{k} \leq H^{-11} \quad\left(C_{10}>0 \text { is an absolute constant }\right),
$$

which is precisely the condition $H \geq C_{0} \log \log \left(T^{k}+100\right)$ of Theorem 1 . We need the condition $H \leq T$ for the bound on $|\zeta(\sigma+i t)|$ mentioned above.

5. Concluding remarks. The new kernel $K(w)$ is very useful. We note that for $|u| \leq 200$ it satisfies the relation

$$
\int_{-\infty}^{\infty}|K(u+i v)| d v=\left(\int_{-\infty}^{\infty} K(i v) d v\right)\left(1+O\left(\frac{1}{A}\right)\right)
$$

(for large $A$ ), which is not hard to verify. Using this we can prove the following theorem.

THEOREM 13. Let $a_{1}, a_{2}, \ldots$ be any infinite sequence of complex numbers and $\lambda_{1}, \lambda_{2}, \ldots$ any sequence of real numbers satisfying $a_{1}=\lambda_{1}=1, \lambda_{n+1}-\lambda_{n}$ bounded both above and below by positive constants, and $\left|a_{n}\right|$ bounded above by a positive constant power of $n$. Suppose that

$$
F(s)=\sum_{n=1}^{\infty} a_{n} \lambda_{n}^{-s}
$$

(which is certainly analytic in a half plane) can be continued in $(\sigma \geq$ $1 / 2, T-H \leq t \leq T+2 H)$ and there satisfies the condition that $M$ defined by $M=\max |F(s)|$ satisfies $\log \log (M+100)=o(T)$. Let $k$ be any positive real number which is less than an absolute (arbitrary) constant. Let $\varepsilon(>0)$ be any constant. Then there exists a constant $C_{11}=C_{11}(\varepsilon)(>0)$ independent of $k$ such that for all $T \geq 2 H \geq C_{11}(\varepsilon) \log \log \left(M^{2 k}+100\right)$, we have

$$
\min _{\sigma \geq 1 / 2}\left(\frac{1}{H} \int_{T}^{T+H}|F(\sigma+i t)|^{2 k} d t\right) \geq 1-\varepsilon .
$$

Proof. We argue as in the proof of Theorem 11 taking $f_{0}(z)=F(z)$, $\sigma_{0}=1 / 2, a$ equal to a large constant depending on $\varepsilon$ and $n=2$. This leads to the proof of theorem on using (18).

The application to the Riemann zeta-function is obvious. It runs as follows. (We use $|\zeta(\sigma+i t)| \ll t^{1 / 2}$ for $\sigma \geq 1 / 2, t \geq 10$.)

THEOREM 14. Let $k$ be any positive number which is bounded above and $\varepsilon(>0)$ any constant. Then there exists a constant $C_{12}(\varepsilon)(>0)$ independent of $k$ such that for all $H$ satisfying $T \geq H \geq C_{12}(\varepsilon) \log \log \left(T^{k}+100\right)$, we 
have

$$
\min _{\sigma \geq 1 / 2}\left(\frac{1}{H} \int_{T}^{T+H}|\zeta(\sigma+i t)|^{2 k} d t\right) \geq 1-\varepsilon .
$$

By taking $H=T$ we recover the following special case.

Theorem 15 (A. Ivić and A. Perelli [3]). We have, for all $k>0$,

$$
\frac{1}{T} \int_{T}^{2 T}|\zeta(1 / 2+i t)|^{2 k} d t \geq 1+o(1)
$$

uniformly in $k$ as $T \rightarrow \infty$.

Remark. The proof of Theorem 15 by Ivić and Perelli is completely different.

Acknowledgements. The author is extremely grateful to the referee for some comments which helped the author to correct some oversights.

\section{References}

[1] D. R. Heath-Brown, Fractional moments of the Riemann zeta-function, J. London Math. Soc. (2) 24 (1981), 65-78.

[2] A. Ivić, Lectures on Mean Values of the Riemann Zeta-Function, Tata Inst. Fund. Res. Lectures on Math. and Phys. 82, Springer, 1991.

[3] A. Ivić and A. Perelli, Mean values of certain zeta-functions on the critical line, Liet. Mat. Rink. 29 (1989), 701-714.

[4] M. Jutila, On the value distribution of the zeta-function on the critical line, Bull. London Math. Soc. 15 (1983), 513-518.

[5] H. L. Montgomery and R. C. Vaughan, Hilbert's inequality, J. London Math. Soc. (2) 8 (1974), 73-82.

[6] K. Ramachandra, On the Mean-Value and Omega-Theorems for the Riemann Zeta-Function, Tata Inst. Fund. Res. Lectures on Math. and Phys. 85, Springer, 1995.

[7] E. C. Titchmarsh, The Theory of the Riemann Zeta-Function, 2nd ed., revised and edited by D. R. Heath-Brown, Clarendon Press, Oxford, 1986.

School of Mathematics

Tata Institute of Fundamental Research

Homi Bhabha Road

Mumbai (Bombay) 400 005, India

E-mail: kram@tifrvax.tifr.res.in
Present address:

N.I.A.S

I.I.Sc. (Campus)

Bangalore 560012, India

E-mail: kram@math.tifrbng.res.in 